\title{
On Isoperimetric Inequalities of Riesz Potentials and Applications
}

\author{
Tynysbek Sh. Kalmenov, Ernazar Nysanov, Bolys Sabitbek \\ Institute of Mathematics and Mathematical Modeling, Almaty, Kazakhstan \\ Email: bolus91.ark@mail.ru
}

Received March 17, 2013; revised April 18, 2013; accepted April 25, 2013

Copyright (C) 2013 Tynysbek Sh. Kalmenov et al. This is an open access article distributed under the Creative Commons Attribution License, which permits unrestricted use, distribution, and reproduction in any medium, provided the original work is properly cited.

\begin{abstract}
In this article, we prove certain isoperimetric inequalities for eigenvalues of Riesz potentials and show some applications of the results to a non-local boundary value problem of the Laplace operator.
\end{abstract}

Keywords: Isoperimetric Inequalities; Eigenvalues of the Laplacian; Riesz Potentials

\section{Introduction}

Historically, the minimization of the first eigenvalue of the Dirichlet Laplacian is probably the first such problem which appeared in the scientific literature. In Rayleigh's famous book "Theory of Sound" [1] (first published in 1877), by using some explicit computation and physical interpretations, he stated that a circle minimizes (among all domains of the same area) the first eigenvalue of the Laplacian with the Dirichlet boundary condition. The proof of this conjecture was obtained only after 30 years later, simultaneously (and independently) by G. Faber and E. Krahn. Nowadays, the Rayleigh-Faber-Krahn inequality has been expanded many other boundary value spectral problems and operators; see [2,3] for further references.

In the present paper, we give simple proofs of some isoperimetric inequalities for the eigenvalues of Riesz potential by using methods of symmetrical decreasing rearrangements of positive measurable functions and variational principles. Riesz potentials, that is convolution operators with fractional powers of the distance to a point in $R^{d}$, have important roles in fractional calculus theory. We also apply these results for the Laplacian with a nonlocal boundary conditions, in particular, we prove Rayleigh-Faber-Krahn inequality for the obtained non-local boundary value spectral problem of the Laplacian.

\section{Main Results}

Let in an open bounded domain $\Omega$ of $R^{d}(d \geq 2)$ the following spectral eigenvalue problem of the Riesz potential has discrete spectrum:

$$
\lambda u(x)=\int_{\Omega} \frac{u(y)}{C_{\alpha}|x-y|^{d-\alpha}} \mathrm{d} y, 0<\alpha<d,
$$

where

$$
|x-y|=\left[\sum_{k=1}^{d}\left(x_{k}-y_{k}\right)^{2}\right]^{\frac{1}{2}}
$$

is the distance between $x=\left(x_{1}, \cdots, x_{d}\right)$ and $y=\left(y_{1}, \cdots, y_{d}\right)$ in the $d$-dimensional Euclidean space $R^{d}, C_{\alpha}=\pi^{\frac{d}{2}} 2^{\alpha} \frac{\Gamma\left(\frac{\alpha}{2}\right)}{\Gamma\left(\frac{d-\alpha}{2}\right)}$ and $\Gamma$ is the gamma func-

tion. The potential $u$ satisfies in the distributional sense ( $\chi_{\Omega}$ is the characteristic function of the set $\Omega$ )

$$
(-\Delta u)^{\frac{\alpha}{2}}=\frac{1}{\lambda} \chi_{\Omega} u \text { in } R^{d} .
$$

Note that when $d \in N$ and $\alpha=2$ Riesz potential coincides with the classical Newtonian potential and for $\alpha=2 m$ satisfies a polyharmonic equation in $R^{d}$ (cf. [4]). We denote eigenvalues of the Reisz potential by $\lambda_{1}(\Omega)>\lambda_{2}(\Omega) \geq \lambda_{3}(\Omega) \geq \cdots \quad$ (enumerate their eigenvalues in decreasing order) each time repeated according to multiplicity. For the spectral problem (2.1), we obtain the following results:

Theorem 1. Let $\Omega \subset R^{d}$ open simple-connected bounded domain and $\Omega^{*} \subset R^{d}$ is a ball of the same measure as the $\Omega$, i.e. $\left|\Omega^{*}\right|=|\Omega|$, then

$$
\lambda_{1}\left(\Omega^{*}\right) \geq \lambda_{1}(\Omega) \text {. }
$$

Theorem 2. Let $\Omega \subset R^{d}$ open simple-connected 
bounded domain and $\Omega^{*} \subset R^{d}$ is a ball of the same measure as the $\Omega$, i.e. $\left|\Omega^{*}\right|=|\Omega|$, and the following series has convergence then

$$
\sum_{i=1}^{\infty} \lambda_{i}^{2}\left(\Omega^{*}\right) \geq \sum_{i=1}^{\infty} \lambda_{i}^{2}(\Omega) .
$$

\section{Preliminary}

Let $\Omega$ bounded measurable set in $R^{d}$. Its symmetric rearrangement $\Omega^{*}$ is an open ball originated at 0 with a volume equal to the volume of $\Omega$, i.e. $\left|\Omega^{*}\right|=|\Omega|$ and

$$
\Omega^{*}=\left\{x \in R^{d}, \quad \omega_{d}|x|^{d}<|\Omega|\right\},
$$

where $\omega_{d}=\frac{2 \pi^{\frac{d}{2}}}{\Gamma\left(\frac{d}{2}\right)}$ is the surface area of the unit sphere in $R^{d}$. Let $u$ be a nonnegative measurable function vanishing at infinity, in the sense that all its positive level sets have finite measure,

$$
\operatorname{Vol}(\{x \mid f(x)>t\})<\infty,(\forall t>0) .
$$

In the definition of the symmetric decreasing rearrangement of $u$ can be used the layer-cake decomposition (see, for example, [5]), which expresses a nonnegative function $u$ in terms of its level sets as

$$
u(x)=\int_{0}^{\infty} \chi_{\{u(x)>t\}} \mathrm{d} t .
$$

where $\chi$ is the characteristic function of the corresponding domain.

Definition. A function

$$
u^{*}(x)=\int_{0}^{\infty} \chi_{\{u(x)>t\}^{*}} \mathrm{~d} t
$$

is called a symmetric decreasing rearrangement of $u$.

As its level sets are open domains $u^{*}$ is lower semicontinuous function, and it is uniquely determined by the distribution function

$$
\mu_{u}(t)=\operatorname{Vol}(\{x \mid u(x)>t\}) .
$$

By construction, $u^{*}$ is equimeasurable with $u$, i.e. corresponding level sets of the two functions have the same volume,

$$
\mu_{u}(t)=\mu_{u^{*}}(t), \quad(\forall t>0) .
$$

Lemma 1. For each non-negative function of $u$ from $H(\Omega)$, we have

$$
\|u\|_{H(\Omega)}=\left\|u^{*}\right\|_{H\left(\Omega^{*}\right)} .
$$

Proof. Using the layer-cake decomposition (3.1), Fubini's theorem and (3.2), we obtain

$$
\begin{aligned}
& \int_{\Omega}|u(x)|^{2} \mathrm{~d} x=\int_{\Omega}^{\infty} \int_{0}^{\infty} \chi_{\left\{u^{2}(x)>t\right\}} \mathrm{d} t \mathrm{~d} x \\
& =\int_{0}^{\infty} \operatorname{Vol}\left(\left\{u^{2}(x)>t\right\}\right) \mathrm{d} t=\int_{0}^{\infty} \operatorname{Vol}(\{u(x)>s\}) 2 s \mathrm{~d} s \\
& =\int_{0}^{\infty} \mu_{u}(s) 2 s \mathrm{~d} s=\int_{0}^{\infty} \mu_{u^{*}}(s) 2 s \mathrm{~d} s \\
& =\int_{0}^{\infty} \operatorname{Vol}\left(\left\{u^{*}(x)>s\right\}\right) 2 s \mathrm{~d} s=\int_{0}^{\infty} \operatorname{Vol}\left(\left\{u^{*^{2}}(x)>t\right\}\right) \mathrm{d} t \\
& =\int_{\Omega}^{\infty} \int_{0}^{\infty} \chi_{\left\{u^{*^{2}}(x)>t\right\}} \mathrm{d} t \mathrm{~d} x=\int u_{\Omega}\left|u^{*}(x)\right|^{2} \mathrm{~d} x .
\end{aligned}
$$

Lemma 1 is proved.

In proofs of the theorems we use the following

\section{F. Riesz's inequality $[5,6]$}

$$
\begin{aligned}
& \int_{\Omega} \int_{\Omega} f(y) g(x-y) h(x) \mathrm{d} y \mathrm{~d} x \\
& \leq \int_{\Omega^{*}} \int_{\Omega^{*}} f^{*}(y) g^{*}(x-y) h^{*}(x) \mathrm{d} y \mathrm{~d} x,
\end{aligned}
$$

where $f^{*}, g^{*}$ and $h^{*}$ symmetric and non-increasing rearrangement of positive measurable functions $f, g$ and $h$ respectively.

\section{Proofs of Theorems 1 and 2}

Since Riesz kernel of the potential (2.1) is symmetric and positive, by Ench theorem [7], its largest eigenvalue $\lambda_{1}$ is positive and simple, and the corresponding eigenfunction $u_{1}(x)$ can be chosen positive in $\Omega$.

Let us use the denotation $\varepsilon_{d, \alpha}(x-y):=\frac{1}{C_{\alpha}|x-y|^{d-\alpha}}$.

Using the Riesz inequality (3.3) and the fact that $\varepsilon_{d, \alpha}(x-y)$ is positive, symmetric and decreasing for all $(x, y) \in \Omega \times \Omega$, we have

$$
\begin{aligned}
& \int_{\Omega} \int_{\Omega} u_{1}(y) \varepsilon_{d, \alpha}(x-y) u_{1}(x) \mathrm{d} y \mathrm{~d} x \\
& \leq \int_{\Omega^{*}} \int_{\Omega^{*}} u_{1}^{*}(y) \varepsilon_{d, \alpha}(x-y) u_{1}^{*}(x) \mathrm{d} y \mathrm{~d} x .
\end{aligned}
$$

Hence by Lemma 1 and the variational principle for $\lambda_{1}\left(\Omega^{*}\right)$, we have

$$
\begin{aligned}
\lambda_{1}(\Omega) & =\frac{\int_{\Omega} \int_{\Omega} u_{1}(y) \varepsilon_{d, \alpha}(x-y) u_{1}(x) \mathrm{d} y \mathrm{~d} x}{\int_{\Omega}\left(u_{1}(x)\right)^{2} \mathrm{~d} x} \\
& \leq \frac{\int_{\Omega^{*}} \int_{\Omega^{*}} u_{1}^{*}(y) \varepsilon_{d, \alpha}(x-y) u_{1}^{*}(x) \mathrm{d} y \mathrm{~d} x}{\int_{\Omega^{*}}\left(u_{1}^{*}(x)\right)^{2} \mathrm{~d} x} \\
& \leq \sup _{v \in H\left(\Omega^{*}\right)} \frac{\int_{\Omega^{*}} \int_{\Omega^{*}} v(y) \varepsilon_{d, \alpha}(x-y) v(x) \mathrm{d} y \mathrm{~d} x}{\int_{\Omega^{*}}(v(x))^{2} \mathrm{~d} x} \\
& =\lambda_{1}\left(\Omega^{*}\right) .
\end{aligned}
$$

Theorem 1 is completely proved. 
Note 1. One may wonder whether the ball is only maximizer of $\lambda_{1}$ among all domains of the same volume. But the answer is no. For example, if we remove a set of zero capacity from the ball, a new domain also maximizes the value of $\lambda_{1}$ since the Hilbert space $H(\Omega)$ does not change if we remove from $\Omega$ a set of zero capicity.

Now we prove Theorem 2. By bilinear decomposition of repeated kernel, we have

$$
\sum_{i=1}^{\infty} \lambda_{i}^{2}(\Omega)=\int_{\Omega} \int_{\Omega}\left|\varepsilon_{d, \alpha}(x-y)\right|^{2} \mathrm{~d} x \mathrm{~d} y
$$

From (3.3) and the fact that $\left|\varepsilon_{d, \alpha}(x-y)\right|^{2}$ is a symmetric and positive decreasing function, we obtain

$$
\int_{\Omega} \int_{\Omega}\left|\varepsilon_{d, \alpha}(x-y)\right|^{2} \mathrm{~d} x \mathrm{~d} y \leq \int_{\Omega^{*}} \int_{\Omega^{*}}\left|\varepsilon_{d, \alpha}(x-y)\right|^{2} \mathrm{~d} x \mathrm{~d} y
$$

According to (4.1),

$$
\begin{aligned}
\sum_{i=1}^{\infty} \lambda_{i}^{2}(\Omega) & =\int_{\Omega} \int_{\Omega}\left|\varepsilon_{d, \alpha}(x-y)\right|^{2} \mathrm{~d} x \mathrm{~d} y \\
& \leq \int_{\Omega^{*}} \int_{\Omega^{*}}\left|\varepsilon_{d, \alpha}(x-y)\right|^{2} \mathrm{~d} x \mathrm{~d} y=\sum_{i=1}^{\infty} \lambda_{i}^{2}\left(\Omega^{*}\right)
\end{aligned}
$$

Theorem 2 is proved.

Note 2. We can generalize Theorem 2 writing in the following form

$$
\sum_{i=1}^{\infty} \lambda_{i}^{n}\left(\Omega^{*}\right) \geq \sum_{i=1}^{\infty} \lambda_{i}^{n}(\Omega)
$$

but obviously, in this case we need some restrictions on $n$ depending on the dimension of the Euclidean space $d$ and $\alpha$.

\section{On Applications of Results for Boundary Value Problems of the Laplacian}

Let $d \geq 3$ and $\alpha=2$. In this case, the Riesz potential coincides with the classical Newton potential, that is, the kernel of the Riesz potential is

$$
\varepsilon_{d}(x-y)=\frac{1}{C_{2}|x-y|^{d-2}}, \quad(d \geq 3)
$$

where $d$ is a natural number.

Lemma 2. For any function $f \in H(\Omega)$, suppf $\subset \Omega$ the Newton potential

$$
u(x)=\int_{\Omega} \varepsilon_{d}(x-y) f(y) \mathrm{d} y
$$

satisfies the boundary condition

$$
\begin{aligned}
& -u(x)+2 \int_{\partial \Omega} \frac{\partial \varepsilon_{d}(x-y)}{\partial n_{y}} u(y) \mathrm{d} s_{y} \\
& -2 \int_{\partial \Omega} \varepsilon_{d}(x-y) \frac{\partial u(y)}{\partial n_{y}} \mathrm{~d} s_{y}=0, \quad x \in \partial \Omega .
\end{aligned}
$$

Conversely, if a function $u \in H^{2}(\Omega)$ satisfies

$$
-\Delta u(x)=f(x), x \in \Omega
$$

and the boundary condition (5.2), then the function $u(x)$ coincides with the Newton potential (5.1), here $\frac{\partial}{\partial n_{y}}$ denotes the outer normal derivative on the boundary.

Proof. Suppose that $u \in C^{2}(\Omega) \cap C^{1}(\bar{\Omega})$. A direct calculation shows that, for any $x \in \Omega$, we have

$$
\begin{aligned}
& u(x)=\int_{\Omega} \varepsilon_{d}(x-y) f(y) \mathrm{d} y \\
& =-\int_{\Omega} \varepsilon_{d}(x-y) \Delta_{y} u(y) \mathrm{d} y=-\int_{\Omega} \Delta_{y} \varepsilon_{d}(x-y) u(y) \mathrm{d} y \\
& +\int_{\partial \Omega}\left(\frac{\partial \varepsilon_{d}(x-y)}{\partial n_{y}} u(y)-\varepsilon_{d}(x-y) \frac{\partial u(y)}{\partial n_{y}}\right) \mathrm{d} S_{y} \\
& =u(x)+\int_{\partial \Omega}\left(\frac{\partial \varepsilon_{d}(x-y)}{\partial n_{y}} u(y)-\varepsilon_{d}(x-y) \frac{\partial u(y)}{\partial n_{y}}\right) \mathrm{d} S_{y},
\end{aligned}
$$

where $\frac{\partial}{\partial n_{y}}=n_{1} \frac{\partial}{\partial y_{1}}+\cdots+n_{d} \frac{\partial}{\partial y_{d}}$ and $n_{1}, \cdots, n_{d}$ are components of the unit normal.

This implies, for $x \in \Omega$, we get

$$
\int_{\partial \Omega}\left(\frac{\partial \varepsilon_{d}(x-y)}{\partial n_{y}} u(y)-\varepsilon_{d}(x-y) \frac{\partial u(y)}{\partial n_{y}}\right) \mathrm{d} S_{y}=0 .
$$

Applying properties of single-layer and double-layer potentials [8] to Formula (5.4) with $x \rightarrow \partial \Omega$, we get

$$
\begin{aligned}
& -\frac{u(x)}{2}+\int_{\partial \Omega}\left(\frac{\partial \varepsilon_{d}(x-y)}{\partial n_{y}} u(y)\right. \\
& \left.-\varepsilon_{d}(x-y) \frac{\partial u(y)}{\partial n_{y}}\right) \mathrm{d} S_{y}=0, \quad x \in \partial \Omega,
\end{aligned}
$$

i.e. (5.5) is a boundary condition for the Newton potential (5.1). Passing to the limit we can easily show that (5.5) remains valid for all $u \in H^{2}(\Omega)$. Thus, the Newton potential (5.1) satisfies the boundary condition (5.2).

Conversely, if the function $g \in H^{2}(\Omega)$ satisfies $-\Delta g=f$ and the boundary condition (5.2), then it coincides with the Newtonian potential (5.1). Indeed, if this is not so, then the function $v=u-g \in H^{2}(\Omega)$, where $u$ is the Newton potential (5.1) satisfies the homogeneous equation $\Delta v=0$ with the boundary condition of

$$
\begin{aligned}
& -\frac{v(x)}{2}+\int_{\partial \Omega}\left(\frac{\partial \varepsilon_{d}(x-y)}{\partial n_{y}} v(y)\right. \\
& \left.-\varepsilon_{d}(x-y) \frac{\partial v(y)}{\partial n_{y}}\right) \mathrm{d} S_{y}=0, \quad x \in \partial \Omega .
\end{aligned}
$$


As above, applying Green's formula to $v \in H^{2}(\Omega)$, we see that

$$
\begin{aligned}
0= & \int_{\Omega} \varepsilon_{d}(x-y) \Delta_{y} v(y) \mathrm{d} y \\
= & -v(x)-\int_{\partial \Omega}\left(\frac{\partial \varepsilon_{d}(x-y)}{\partial n_{y}} v(y)\right. \\
& \left.-\varepsilon_{d}(x-y) \frac{\partial v(y)}{\partial n_{y}}\right) \mathrm{d} S_{y}, \quad \forall x \in \Omega .
\end{aligned}
$$

Passing the limit as $x \rightarrow \partial \Omega$, we obtain

$$
\begin{aligned}
& -v(x)+\frac{v(x)}{2}-\int_{\partial \Omega}\left(\frac{\partial \varepsilon_{d}(x-y)}{\partial n_{y}} v(y)\right. \\
& \left.-\varepsilon_{d}(x-y) \frac{\partial v(y)}{\partial n_{y}}\right) \mathrm{d} S_{y}=0, \quad x \in \partial \Omega .
\end{aligned}
$$

From (5.6), it implies

$$
v(x) \mid=0, x \in \partial \Omega .
$$

By virtue of the uniqueness of a solution of the Dirichlet problem for the Laplace equation, we have $v(x)=u(x)-g(x)=0$ for all $x \in \Omega$, that is, $g \equiv u$, $g$ coincides with the Newton potential. This completes the proof of Lemma 2.

Lemma 2 shows that the eigenvalue problem of the Newton potential is equivalent to spectral boundary problem for the Laplace equation

$$
\begin{aligned}
& -\Delta u=\mu u, x \in \Omega, \\
& -u(x)+2 \int_{\partial \Omega} \frac{\partial \varepsilon_{d}(x-y)}{\partial n_{y}} u(y) \mathrm{d} S_{y} \\
& -2 \int_{\partial \Omega} \varepsilon_{d}(x-y) \frac{\partial u(y)}{\partial n_{y}} \mathrm{~d} S_{y}=0, x \in \partial \Omega .
\end{aligned}
$$

Finally, by Theorem 1, we obtain Rayleigh-FaberKrahn inequality for the first eigenvalue $\mu_{1}=\frac{1}{\lambda_{1}}$ of the Laplacian boundary value problems (5.8) and (5.9).

Proposition 1. A ball minimizes the first eigenvalue of the Laplacian (5.8) with a special type of non-local boundary condition (5.9) among all domains of the same measure.
And from Theorem 2 we obtain the following analogue of Dittmar's result [9].

Proposition 2. Series made up of squares of reciprocal eigenvalues of the Laplacian with the boundary condition (5.9),

$$
\sum_{i=1}^{\infty} \frac{1}{\mu_{i}^{2}}
$$

is minimized in a ball among all domains of the same measure.

Note that in [10] in the case of two-dimensional ball and three-dimensional ball we calculated all eigenvalues of the the Laplacian with the boundary condition (5.9).

\section{REFERENCES}

[1] J. W. Rayleigh, "The Theory of Sound," Dover Publishing, New York, 1945.

[2] A. Henrot, "Extremum Problems for Eigenvalues of Elliptic Operators," Birkhauser, Basel, 2006.

[3] D. Daners, "A Faber-Krahn Inequality for Robin Problems in Any Space Dimension," Mathematische Annalen, Vol. 335, 2006, pp. 767-785. doi:10.1007/s00208-006-0753-8

[4] T. Sh. Kalmenov and D. Suragan, "Boundary Conditions for the Volume Potential for the Polyharmonic Equation," Differential Equations, Vol. 48, No. 4, 2012, pp. 595-599.

[5] A. Burchard, "A Short Course on Rearrangement Inequalities," 2009. www.math.toronto.edu/almut/rearrange.pdf

[6] F. Riesz, "Sur Une Inregalitre Intregrale," Journal of the London Mathematical Society, Vol. 5, No. 3, 1930, pp. 162-168. doi:10.1112/jlms/s1-5.3.162

[7] B. S. Vladimirov, "Equations of Mathematical Physics," Nauka, Moscow, 1981.

[8] N. S. Landkoff, "Foundations of Modern Potential Theory," Springer-Verlag, Berlin, 1972. doi:10.1007/978-3-642-65183-0

[9] B. Dittmar, "Sums of Reciprocal Eigenvalues of the Laplacian," Mathematische Nachrichten, Vol. 237, No. 1, 2002, pp. 45-61. doi:10.1002/1522-2616(200204)237:1<45::AID-MANA4 5>3.0.CO;2-M

[10] T. Sh. Kalmenov and D. Suragan, "To Spectral Problems for the Volume Potential," Doklady Mathematics, Vol. 428, No. 1, 2009, pp. 16-19. 